\title{
VALIDACIÓN DE UN INSTRUMENTO PARA MEDIR EL NIVEL DE SATISFACCIÓN DE MUJERES EMBARAZADAS DURANTE EL PARTO
}

\author{
VALIDATION OF AN INSTRUMENT TO MEASURE THE LEVEL OF SATISFACTION OF \\ PREGNANT WOMEN DURING THE DELIVERY \\ Jhony A. De La Cruz -Vargas 1,2, Sarita Rodríguez-Chávez,3, Luis Roldan-Arbieto5,6, Alejandro Medina-Vilca², \\ Manuel Huamán-Guerrero' y Miguel Perez ${ }^{1,7}$
}

\begin{abstract}
RESUMEN
Objetivos: Evaluar la validez y fiabilidad de un instrumento adaptado a partir de la escala SERVPERF para medir el nivel de satisfacción en gestantes durante el parto en una Institución de Salud en Lima, Perú. Métodos: Estudio transversal, descriptivo con componentes analíticos de validación del cuestionario SERVPERF adaptado para mujeres gestantes peruanas (GP) durante el parto. Se realizó una prueba piloto con posterior análisis de propiedades psicométricas de validez y fiabilidad mediante análisis factorial exploratorio de componentes principales y cálculo de alfa de Cronbach. Resultados: Se reporta una muestra de 345 mujeres, atendidas en la Clínica Good Hope, en el período de febrero a abril del año 2016. La prueba de adecuación de Kaiser-Meyer Olkin fue de 0.919 y la prueba de esfericidad de Barlett de 4008.9 ( $p<0.000)$. La varianza acumulada explicada por los 5 factores fue de $65.039 \%$, La fiabilidad mostró un alfa de Cronbach de 0.92 . Se clasificaron 5 factores con 22 items, redefiniendose un factor como "calidad clinica". Conclusión: El instrumento adaptado y validado SERVPERF-GP posee suficientes propiedades psicométricas para ser considerado una herramienta útil y fiable para medir la satisfacción de gestantes durante su experiencia de parto en instituciones privadas de salud y con potencial aplicación a gestantes en los diversos sectores de salud del Perú.
\end{abstract}

Palabras clave: Validación; Satisfacción; Parto; SERVPERF. (fuente: DeCS BIREME).

\begin{abstract}
Objective: To evaluate the validity and reliability of an adapted instrument from SERPVPERF scale to measure the satisfaction level in pregnant women during childbirth in a Health institution in Lima, Perú. Methods: Cross sectional study, descriptive, with analytical components for SERVPERF questionnaire validation adapted for peruvian pregnant women during childbirth. A pilot test is been performed with subsequent analysis of psychometric properties of validation and reliability, through exploratory factorial analysis of main components and Cronbach's Alpha calculation. Results: It is reported a sample of 345 women treated at Good Hope Clinic between February and April in 2016. The Kaiser Meyer Olkin's adequacy test was 0.919 and the Barlett's sphericity test was $4008.9(p<0.000)$. The cumulative variance explained for the 5 factors was $65.039 \%$. The reliability showed Cronbach's Alpha 0.92. There were classified 05 factors with 22 items so a factor was redefined as "Clinical quality". Conclusions: This adapted and validated SERVPERF-GP instrument gathers sufficient psychometrical properties and should be considered as a reliable and useful measurement tool for pregnant women satisfaction during childbirth experience in Private Health Institutions and with potential application in different Health Sectors in Peru.
\end{abstract}

Key words: Validation; Satisfaction; Childbirth; SERVPERF. (source: MeSH NLM).

\footnotetext{
'Instituto de Investigación en Ciencias Biomédicas (INICIB), Facultad de Medicina Humana, Universidad Ricardo Palma (FAMURP). Lima, Perú.

2 Departamento de Docencia e Investigación, Clínica Good Hope, Lima, Perú.

3 Departamento de Ginecología y Obstetricia, Clínica Good Hope. Lima, Perú.

${ }^{4}$ Maestría en Gerencia de Servicios de Salud, Universidad Nacional Mayor de San Marcos, Lima, Perú.

Unidad de Bioestadística, INICIB. FAMURP, Lima, Perú.

${ }^{6}$ Escuela de Post-Grado de la Universidad San Ignacio de Loyola.

7 Universidad Estatal de Califtonia, Fresno.
}

Correspondencia: Prof. Jhony A. De La Cruz Vargas. Dirección: INICIB, Facultad de Medicina Humana, Edificio I. 2do piso. Avenida Benavides 5440, Surco. Lima, Perú. Teléfono: 708-0000 Anexo: 6016. Correo : jhony.delacruzv@urp.pe

Citar como: Jhony A. De La Cruz -Vargas, Sarita Rodríguez-Chávez, Luis Roldan-Arbieto, Alejandro Medina-Vilca, Manuel Huamán-Guerrero y Miguel Perez. Validación de un instrumento para medir el nivel de satisfacción de mujeres embarazadas durante el parto [Artículo Original]. Rev. Fac. Med. Hum. 2016;16(3):30-37. DOI 10.25176/RFMH.v16.n3.650 


\section{INTRODUCCIÓN}

La metodología para mejorar la "Calidad de la Asistencia Sanitaria" empezó a aplicarse en los años 50 en Estados Unidos. En 1951, la "Joint Commission on Acreditation of Hospitals"1 planteó los parámetros de calidad que los hospitales debían cumplir para ser acreditados y en 1966, A. Donabedian plantea los principios de la calidad asistencial en base a criterios de estructura, proceso y resultados ${ }^{2}$. Así se establecen las bases de los sistemas de calidad que debian aplicarse a la asistencia en salud, que han ido actualizándose con aportaciones normativas basadas en modelos de calidad como ISO ${ }^{3}$ y EFQM (European Foundation for Quality Management, EFQM) ${ }^{4-6}$, o los criterios de acreditación aplicados a cada uno de los niveles asistenciales de la Joint Commission? ${ }^{7}$.

La calidad de los servicios sanitarios clásicamente se ha evaluado mediante los resultados directos e indirectos de la atención sanitaria desde la óptica de los profesionales de salud. Sin embargo, Para evaluar la calidad de los resultados en salud, resulta fundamental e imprescindible incorporar el punto de vista del usuario a través de estudios de satisfacción ${ }^{8}$. Autores como Donabedian ${ }^{2}$ y Ware $^{9}$ han mostrado lo importante y pertinente que es la introducción de la satisfacción de los usuarios con los servicios de salud (calidad percibida) como componente esencial y complementaria de otras actividades de evaluación y mejora de la calidad asistencial ya que, sólo conociendo las expectativas y necesidades sentidas por los pacientes-usuarios, se puede proporcionar una correcta orientación de los servicios prestados. El paciente evalúa la calidad de manera diferente al profesional, valorando no solo el resultado final, sino ademas en función de cómo percibe el servicio brindado.

Diversas manera para evaluar el nivel de satisfacción del usuario con el sistema sanitario han sido descritos, como el método de quejas y sugerencias ${ }^{10}$, o estudios con metodología cualitativa ${ }^{11}$. Sin embargo, en países de latinoamérica, es usual evaluar la satisfacción de los servicios de salud, mediante cuestionarios o encuestas de satisfacción ${ }^{12}$.

Las escalas de evaluación son aquellos instrumentos que permiten un escalamiento acumulativo de sus ítems, dando puntuaciones globales al final de la evaluación. Su carácter acumulativo las diferencia de los cuestionarios de recogida de datos, las entrevistas estandarizadas o los formularios ${ }^{13}$.

Las encuestas de satisfacción en salud, a los pacientes deben reunir una serie de propiedades psicométricas que garanticen tanto su fiabilidad como su validez, y que nos permitan valorar la adecuación de la escala al fenómeno objeto de la medición y la calidad de la medida. Durante los últimos años se han desarrollado numerosos cuestionarios para medir el nivel de satisfacción del usuario con la atención sanitaria ${ }^{14-16}$, pero no todos ellos han sido validados, lo que reduce la posibilidad de extrapolar y de comparar resultados. Esto, junto al hecho de que algunas encuestas desarrolladas y validadas en entornos geográficos distintos al nuestro pueden ser difícilmente adaptables debido a diferencias culturales, lingüísticas o en las características del Sistema Sanitario, originando la necesidad de contar con encuestas de satisfacción que hallan sido validadas en nuestro entorno para conocer la calidad percibida por los usuarios, detectar áreas de mejora, extrapolar resultados y permitir la comparación entre diferentes centros.

La satisfacción de las mujeres durante su experiencia de parto es un concepto complejo y multidimensional.

En este estudio elegimos el modelo SERVPERF, cuyo nombre es debido a la exclusiva atención que presta a la valoración del desempeño (SERVice PERFormance) para medir la calidad de servicio. Estructuralmente tiene los mismos ítems y dimensiones que el SERVQUAL (SERVice QUALity), teniendo como única diferencia que la parte que hace referencia a las expectativas de los clientes, es eliminada. De manera interesante quienes elaboraron el modelo SERVPERF, Cronin y Taylor ${ }^{17,18}$, llegaron a la conclusion de que el modelo SERVQUAL de Calidad de Servicio, parece no ser el mas adecuado al momento de evaluar la Calidad del Servicio.

El objetivo principal de este trabajo es evaluar la validez y fiabilidad de un instrumento adaptado a partir de la escala SERVPERF para medir el nivel de satisfacción en gestantes peruanas durante el parto en una Institución de Salud en Lima, Perú.

\section{MÉTODOS}

Diseño: diseñamos un estudio transversal, descriptivo con componentes analíticos de adaptación y validación del cuestionario SERVPERF para mujeres gestantes durante el parto.

Sujetos: La población de estudio fueron las mujeres embarazadas atendidas en el "Departamento de Gíneco-Obstetricia" de la Clínica Good Hope (CGH) donde se atienden aproximadamente 1,776 nacimientos por año (datos del 2015, libro registro de 
nacimientos). El cálculo del tamaño de la muestra se realizó, tomando en cuenta una precisión del 4\%, un nivel de confianza del 95\%, una tasa de no respuesta de $10 \%$ y un porcentaje esperado de satisfacción del $80 \%$, obteniendose 347 pacientes. El estudio se realizó durante el mes de febrero-abril de 2016 y contó con la aprobación previa por el Comité de Docencia e Investigación de la Clínica Good Hope.

\section{Adaptación del cuestionario}

La adaptación del cuestionario fue realizada por un grupo de expertos de carácter multidisciplinario de 5 miembros, formado por profesionales médicos y de obstetricia, de la Jefatura de Docencia e Investigación de la Clínica Good Hope y del Instituto de Investigación en Ciencias Biomédicas de la Universidad Ricardo Palma. Se desarrolló la primera versión del Cuestionario SERVPERF-GP 1.0 (SERVPERF-GESTANTES PERUANAS), el cual evalúa cinco dimensiones de la calidad de servicio recibido por medio de veintidós preguntas, este instrumento contiene una escala de evaluación tipo Likert de 6 puntos que va desde Satisfacción Amplia (6 puntos) hasta Insatisfacción Severa (1 punto). Cada experto evaluó el contenido de los ítems propuestos a fin de cotejarlos de manera cualitativa con los criterios propuestos relativos a relevancia o congruencia con el contenido, claridad en la redacción, tendenciosidad o sesgo en su formulación y dominio del contenido. Esta primera versión del instrumento presentada a "Juicio de Expertos" mostró observaciones a las preguntas 3 y 4. La pregunta 3: ¿ ¿Existe dificultad para entender las preguntas del instrumento?" y la pregunta 4: "¿Existen palabras difíciles de entender en los ítems o reactivos del instrumento?" correspondientes a las preguntas del Cuestionario SERVPERF-GP a adaptar. Estas fueron reajustadas, alcanzando finalmente el 100\% de aprobación de los ítems, por lo que se infiere que la evaluación inicial de contenido adaptado a gestantes peruanas fue satisfactorio.

\section{Prueba Piloto}

La prueba piloto se llevó a cabo en el mes de enero del 2016. Posterior al juicio de expertos, se aplicó el instrumento SERVPERF-GP versión 2.0 a un grupo de usuarias, teniendo en cuenta los criterios de selección (mujeres gestantes con atención de su parto en nuestra Institución de Salud, con recién nacido en alojamiento conjunto y que acepten participar del estudio). Se incluyó un total de 30 usuarias, que luego de leer y aceptar el consentimiento informado, participaron de manera voluntaria. Solo una de ellas manifestó duda con respecto a una pregunta, la que posteriormente fue corregida, elaborándose la versión final del cuestionario. Finalmente el instrumento adaptado versión 3.0 cuenta con 22 ítems corregidos y agrupados en 5 dimensiones. En la primera dimensión, aspectos tangibles están las preguntas 1 a 3 ; en capacidad de respuesta 4 a 6, en empatía 7 a 10, en seguridad 11 a 15 y en confiabilidad de 16 a 22.

La encuesta fue realizada de febrero-abril de 2016 por el equipo profesional del Servicio de Obstetricia, que habitualmente está en contacto directo desde el ingreso de las mujeres embarazadas. La encuesta fue aplicada antes del alta de la paciente y fue contestada por ella misma y de ser necesario se le brindaba asistencia o ayuda. La administración de las encuestas fue diariamente y los datos recogidos fueron guardados en una base de datos Excel y analizadas en el programa informático SPSS 22.0 Adicionalmente se incluyeron preguntas sociodemográficas.

Análisis de la validez del cuestionario: Se utilizó la técnica del Análisis Factorial Exploratorio de Componentes Principales ${ }^{19}$, para evaluar la validez de constructo, luego con la finalidad de alcanzar una mejor comprension de la matriz resultante se aplico la rotación de Varimax. La medida de adecuación muestral de Kaiser-Meyer-Olkin (KMO), el Test de esfericidad de Barlett y el determinante de la matriz de correlaciones, fueron utilizados para comprobar los supuestos de aplicación ${ }^{19}$.

La fiabilidad se evaluó mediante el coeficiente Alfa de Cronbach ${ }^{20}$, para todo el cuestionario y para cada uno de los factores de forma independiente, calculándose también la correlación ítem- total corregido y el valor alfa de Cronbach si el ítem es eliminado.

\section{RESULTADOS}

El presente estudio reporta una muestra de 345 mujeres, puérperas de parto vaginal y parto por cesárea, atendidas en la Clínica Good Hope, en el período de febrero a abril del año 2016. Tres mujeres se negaron a completar la encuesta por motivos de tiempo. En la Tabla 1 se muestra las características generales y sociodemográficas de las usuarias.

\section{Validez de Constructo}

Para la validación del instrumento, se efectuó un análisis factorial exploratorio de componentes principales con los 22 ítems, previamente se verificó su viabilidad a través de las correlaciones bivariadas significativas, se obtuvo un índice KMO de 0.919 y una prueba de esfericidad de Barlett de 4008.9 ( $p<0.000)$. La varianza explicada por los 5 factores obtenidos fue $65.039 \%$, cuyos autovalores se encontraron por encima de uno (Tabla 2), Análisis factorial. 
En la Tabla 3 Matriz de componentes, se muestra la asignación de los ítems a cada uno de los cinco factores, el cual se hizo a través de las cargas factoriales, para lo cual se realizó una rotación Varimax de los factores, con el objetivo de redistribuir las cargas factoriales y facilitar su clasificación, sin afectar la proporción de la varianza explicada. Para la asignación de cada ítem se consideraron valores superiores a 0.5 , sin embargo dos ítems (8 y 16) con valores limitrofes fueron reconsiderados debido al contenido que encierran dichas preguntas.

\section{Confiabilidad}

Se analizó la fiabilidad mediante evaluando la consistencia interna mediante el coeficiente Alfa de Cronbach, obteniéndose un valor de 0.92 (tabla 4).

Versión final del cuestionario SERVPERF-GP 3.0: (Satisfacción del Usuario Gestantes Peruanas)

Producto del proceso de validación se obtuvo un cuestionario final (anexo 1) compuesto por 20 ítems que determinan 5 dimensiones o factores. La Tabla 5 refleja la propuesta de adaptación del instrumento de nuestro grupo de investigación.

Tabla 1. Características generales.

\begin{tabular}{|c|c|c|}
\hline Edad (Rango de 14 a 42) & $\mathbf{N}^{\circ}$ & $\%$ \\
\hline Promedio & $31.5+/-4.95$ & - \\
\hline Menor o igual a 18 & 2 & 0.6 \\
\hline $19-28$ & 103 & 29.9 \\
\hline 29-38 & 216 & 62.6 \\
\hline $39 a+$ & 24 & 7 \\
\hline \multicolumn{3}{|l|}{ Grado de instrucción } \\
\hline Primaria & 1 & 0.3 \\
\hline Secundaria & 16 & 4.6 \\
\hline Superior (pregrado) & 287 & 83.2 \\
\hline Post Grado & 41 & 11.9 \\
\hline \multicolumn{3}{|l|}{ Estado Civil } \\
\hline Soltera & 34 & 9.9 \\
\hline Casada & 205 & 59.4 \\
\hline Conviviente & 102 & 29.6 \\
\hline Divorciada & 4 & 1.2 \\
\hline \multicolumn{3}{|l|}{ Tipo de Parto } \\
\hline Por Cesárea & 270 & 78.3 \\
\hline Vaginal & 75 & 21.7 \\
\hline \multicolumn{3}{|l|}{ Tipo de Cobertura de Atención } \\
\hline Sistema Privado EPS* & 272 & 78.8 \\
\hline Seguro Good Hope & 53 & 15.4 \\
\hline Pago Particular & 20 & 5.8 \\
\hline \multicolumn{3}{|l|}{ Número de Hijos (Rango de 1 a 4) } \\
\hline Promedio & $1.58+/-0.68$ & - \\
\hline 1 & 181 & 52.5 \\
\hline 2 & 128 & 37.1 \\
\hline 3 & 35 & 10.1 \\
\hline 4 & 1 & 0.3 \\
\hline
\end{tabular}

*Entidad Prestadora de Salud: RIMAC, PACIFICO, MAPFRE, LA POSITIVA Fuente: Grupo de estudios INICIB - Good Hope 
Tabla 2. Análisis factorial.

\begin{tabular}{ccccc} 
Componente & Autovalores & \% de la varianza & \% acumulado & Total \\
1 & 9.0087 & 41.306 & 41.306 & 9.087 \\
\hline 2 & 2.011 & 9.142 & 50.448 & 2.011 \\
\hline 3 & 1.320 & 6.000 & 56.448 & .920 \\
\hline 4 & 987 & 4.488 & 60.936 & .903 \\
\hline
\end{tabular}

Fuente: Grupo de estudios INICIB - Good Hope

Tabla 3. Matriz de componente.

\begin{tabular}{|c|c|c|c|c|c|}
\hline \multirow[b]{2}{*}{ Ítem } & \multicolumn{5}{|c|}{ Componente } \\
\hline & 1 & 2 & 3 & 4 & 5 \\
\hline Ítem 10 & .790 & .192 & .067 & .229 & 2.111 \\
\hline Ítem 6 & .778 & .100 & .224 & .157 & -.002 \\
\hline Ítem 5 & .776 & .047 & .204 & .113 & .257 \\
\hline Ítem 15 & .711 & .181 & .353 & .154 & -.212 \\
\hline Ítem 7 & .74 & .278 & .180 & .253 & .142 \\
\hline Ítem 9 & .663 & .237 & .155 & .184 & .169 \\
\hline Ítem 14 & .560 & .157 & .346 & .334 & .073 \\
\hline Ítem 11 & .492 & .182 & .573 & .282 & .070 \\
\hline Ítem 8 & $.449^{*}$ & .278 & .211 & .430 & .136 \\
\hline Ítem 22 & .388 & .575 & .389 & .102 & -.074 \\
\hline Ítem 12 & .349 & .173 & .667 & .114 & .240 \\
\hline Ítem 16 & .345 & $.453^{*}$ & .209 & .299 & .065 \\
\hline Ítem 13 & .343 & .126 & .752 & .215 & .123 \\
\hline Ítem 21 & .295 & .594 & .474 & .035 & .010 \\
\hline Ítem 4 & .233 & .095 & .134 & .103 & .872 \\
\hline Ítem 1 & .208 & .054 & .055 & .759 & -.085 \\
\hline Ítem 3 & .201 & .064 & .069 & .725 & .036 \\
\hline Ítem 2 & .173 & .074 & .153 & .793 & .185 \\
\hline Ítem 19 & .164 & .670 & .262 & .071 & -.067 \\
\hline Ítem 17 & .124 & .757 & -.122 & .181 & .116 \\
\hline Ítem 18 & .075 & .761 & .128 & -.022 & .136 \\
\hline Ítem 20 & .073 & .504 & .577 & -.004 & -.097 \\
\hline
\end{tabular}

Método de extracción: Análisis de componentes principales.

Método de rotación: Normalización Varimax con Kaiser.

*. Se ha considerado un valor superior a 0.5 para asignarlo a un factor.

Pág. 34 
Tabla 4. Confiabilidad del instrumento.

\begin{tabular}{|c|c|c|}
\hline Dimensión & $\begin{array}{l}\text { Alfa de } \\
\text { Cronbach }\end{array}$ & Ítems \\
\hline $\begin{array}{l}\text { Aspectos } \\
\text { Tangibles }\end{array}$ & 0.72 & 3 \\
\hline Seguridad & 0.86 & 5 \\
\hline $\begin{array}{l}\text { Capacidad de } \\
\text { respuesta }\end{array}$ & 0.71 & 3 \\
\hline $\begin{array}{l}\text { Confiabilidad o } \\
\text { fiabilidad }\end{array}$ & 0.83 & 7 \\
\hline Empatía & 0.71 & 3 \\
\hline $\begin{array}{l}\text { Satisfacción } \\
\text { Global }\end{array}$ & 0.92 & 22 \\
\hline \multicolumn{3}{|c|}{ Fuente: Grupo de estudios INICIB - Good Hope } \\
\hline \multicolumn{3}{|c|}{ Tabla 5. Versión final: SERVPERF-GP. } \\
\hline Factor & $\begin{array}{l}\text { Nombre } \\
\text { del factor }\end{array}$ & Ítems \\
\hline 1 & Calidad Clínica* & $\begin{array}{c}10,6,5,15,7,9 \\
14,8\end{array}$ \\
\hline 2 & Confiabilidad & $\begin{array}{c}21,22,17,18,19 \\
16\end{array}$ \\
\hline 3 & Seguridad & $11,12,13,20$ \\
\hline 4 & $\begin{array}{l}\text { Aspectos } \\
\text { Tangibles }\end{array}$ & $1,2,3$ \\
\hline 5 & $\begin{array}{l}\text { Capacidad de } \\
\text { Respuesta }\end{array}$ & 4 \\
\hline
\end{tabular}

*Calidad clínica: Empatía + capacidad de respuesta

Fuente: Grupo de estudios INICIB - Good Hope

\section{DISCUSIÓN}

En la toma de decisiones tanto en gestión y políticas de salud, en la practica asistencial y en investigación clínica, medir la satisfacción de los pacientes resulta esencial y necesario ${ }^{21,22}$.

Evaluar la calidad de atención del parto ha tomando importancia creciente en las últimas décadas. Las peculiaridades que presenta la atención en los servicios hospitalarios de obstetricia, hacen necesario disponer de una encuesta de satisfacción específica, capaz de describir las opiniones de los pacientes y de identificar problemas susceptibles de mejora.

En nuestro estudio la primera pregunta que nos formulamos fue "¿existe un cuestionario desarrollado y/o validado en nuestro medio para medir específicamente la satisfacción de las mujeres embarazadas durantesu parto hospitalario, queexplore los aspectos de la satisfacción que consideramos de interés?". No fue posible encontrar un cuestionario específico y validado en nuestro medio que recogiera dichos aspectos. Por este motivo, se procedió a la adaptación y posterior validación del cuestionario.

La validación de contenido del cuestionario diseñado en nuestro estudio se realizó mediante consenso de expertos utilizando la técnica Delphi ${ }^{23}$. Esta técnica cualitativa aporta la ventaja de conseguir un consenso manteniendo la máxima autonomía de los participantes. La selección de los participantes en la técnica se realizó incorporando expertos en calidad, investigación y profesionales del área clínica implicada con el objetivo de asegurar que todos los aspectos de la atención que influyen en la satisfacción se encuentran incluidos en el cuestionario.

Para realizarla validación de constructo del cuestionario e identificar su estructura factorial utilizamos la técnica de análisis factorial exploratorio de componentes principales. Autores como Batista- Foguet ${ }^{24}$ consideran que el análisis factorial exploratorio no es suficiente para asegurar la validez de un cuestionario enfocado a salud y proponen el análisis factorial confirmatorio como alternativa adecuada ${ }^{25}$. Sin embargo, el análisis factorial exploratorio permite la descripción y el resumen de los datos agrupando variables que están correlacionadas, sin necesidad de conocer los factores a priori, en contraposición con el análisis factorial confirmatorio que requiere que las variables hayan sido cuidadosamente elegidas para revelar el factor subyacente. En nuestro estudio hemos preferido utilizar el análisis factorial exploratorio como herramienta para reducir el número de variables y examinar pautas de correlación entre ellas, sin un intento riguroso de elaborar teorías, y partiendo de un punto de partida puramente especulativo.

Posterior al análisis factorial y la extracción del menor número de factores que explicaban estas correlaciones, se realizó una rotación Varimax de la solución para conocer en profundidad las variables más relacionadas con cada factor. Batista- Foguet ${ }^{25}$ consideran la rotación un método arbitrario que puede conducir a interpretaciones distintas. Sin embargo, en nuestro estudio decidimos utilizar la rotación Varimax para facilitar la comprensión de la matriz resultante e identificar claramente las variables relacionadas con cada factor.

En nuestro estudio, la fiabilidad se ha medido, utilizando el coeficiente Alfa de Cronbach. La fiabilidad entendida como estabilidad temporal o fiabilidad testretest, y la concordancia interobservadores no fueron 
determinadas en este estudio, ya que para esto sería preciso "medir"y "volver a medir", situación no practica en la población evaluada y debido a las características del cuestionario (autocumplimentado y anónimo).

La fiabilidad y la validez constituyen el binomio fundamental para la medición y son los indicadores de calidad de los cuestionarios. La fiabilidad tiene un carácter eminentemente practico y explica el rendimiento de las mediciones realizadas, y la validez se enfoca en la parte teorica a la pregunta ¿para que es valido el instrumento?.

La fiabilidad expresada por el valor Alfa de Cronbach y la validez por el análisis factorial exploratorio garantizan la validación de los cuestionarios, el análisis de la validez debe preceder al de la fiabilidad, como se ha realizado en nuestro estudio, debido a que de no seria util una medida fiable de algo diferente a lo que se deseamos medir. El proceso de recogida de datos fue homogéneo, y de auto Ilenado. El porcentaje de rechazos a completar la encuesta fue mínimo.

En muchas instituciones de salud de Perú y Latinoamérica, la satisfacción del usuario se evalúa basado en un sistema de quejas que presentan los pacientes o familiares, encuestas simples y encuestas de satisfacción tipo SERVQUAL. Múltiples estudios realizados en el Perú sobre la calidad de la atención en el parto, Oviedo Sarmiento (2010)26, Lora Loza $(2006)^{27}$, Wajajay Nuñez $(2013)^{28}$, Espinoza Rojas $(2014)^{29}$, Miranda Benavente y Cols. 2006) ${ }^{30}$ muestran que existen diversos niveles de satisfacción, más allá de las heterogéneas poblaciones estudiadas, diversos contextos y diferentes metodologías y herramientas empleadas.

EI SERVPERF original propone 22 ítems que fueron utilizados, sin embargo, en nuestro estudio hallamos que los ítems 8 y 16 mostraban una carga factorial baja, de bajo poder de discriminación para la asignación a uno de los 5 factores identificados. El instrumento final propuesto podría estar conformado por 20 ítems, agrupados en cinco dimensiones, pero esto necesita ser confirmado en futuros estudios, por lo que mantenemos la versión final con 22 items. Otro alcance de nuestro estudio es que el factor Empatía, recibió asignación de items correspondientes al factor Seguridad, proponiendo nuestro grupo el nombre del nuevo factor combinado como: Calidad Clinica.

Resulta evidente enfatizar que la población estudiada que corresponde al sistema privado del Perú, posee características propias como nivel socioeconómico, grado de instrucción, edad media, numero de hijos, estado civil, tipo de parto, sistema de cobertura de atención, etc. Por lo que hemos iniciado el proceso de validación de este cuestionario para los hospitales públicos del país.

El trabajo que presentamos aporta un cuestionario adaptado específicamente para conocer la satisfacción de las mujeres embarazadas durante su experiencia de parto, tras el proceso de validación, la versión final SERVPERF-GP ha demostrado reunir las propiedades métricas necesarias para poder ser considerado un instrumento útil y fiable, de forma que podemos recomendar su utilización. Presenta las ventajas de un número reducido de ítems, una escala de respuesta sencilla que es fácil de entender por las pacientes y puede responderse en un tiempo corto. Las características de nuestro estudio, que aborda el ámbito hospitalario privado del Perú, nos permite considerarlo de referencia en el ámbito de las encuestas de satisfacción para mujeres embarazadas.

\section{CONCLUSIÓN}

El instrumento adaptado y validado SERVPERPF-GP posee propiedades psicométricas suficientes para ser considerado una herramienta útil y fiable para medir la satisfacción de gestantes durante su experiencia de parto en instituciones privadas de salud y con potencial aplicación a mujeres gestantes en los diversos subsectores de salud del Perú.

\section{Agradecimientos}

A la Dra. Norca Huamalies Baquerizo, Directora General de la Clínica Good Hope, por todo el apoyo y las facilidades para realizar este trabajo.

Al equipo de Ginecología y Obstetricia de la Clínica Good Hope, por toda la colaboración recibida.

Financiamiento: Autofinanciado.

Conflicto de interés: Los autores declaran no tener conflictos de interés en la publicación de este artículo.

Recibido: 10 de noviembre

Aprobado: 20 de diciembre 


\section{REFERENCIAS BIBLIOGRÁFICAS}

1. Joint Comission. Estándares de acreditación de hospitales. Barcelona: E: FAD. 1997.

2. Donabedian A. Evaluating the quality of medical care. The Milbank memorial fund quarterly. 1966; 44 (3):166-206.

3. ISO 9001: 2000 - Sistemas de Gestión de Calidad. Requisitos [Internet]. www.chospab.es/calidad/archivos/Documentos/ Normalnternacionallso9001.pdf.

4. EFQM. Directrices para el sector público: salud. Madrid: Club Gestión de Calidad. 1996.

5. Clemente P, Fortuna B, Tort-Martorell X. Modelo de excelencia de EFQM

aplicado al ámbito sanitario. Barcelona: Faura Casas Editorial. 2003.

6. European Foundation for Quality Management. European Excellence Model. Brussels. 2000

7. Joint Commision. Estándares de acreditación de Hospitals. Barcelona: FAD; 1998

8. Thomas R. Zastowny, Klaus J. Roghmann and Gail L. Cafferata. Patient Satisfaction and the Use of Health Services: Explorations in Causality on JSTOR. Medical Care. 1989; 27:705-23.

9. Ware JE Jr, Davies-Avery A, Stewart AL. The measurement and meaning of patient satisfaction. Health Med Care Service Review; 1(1):13-15. 1978

10. Pascual L, Uris J, Alfonso MD, Sanmartín D, Sanz J, Campos C. Las reclamaciones y las hojas de sugerencias como método de incorporación de la opinión del usuario en atención primaria. Comparación con la perspectiva de los profesionales. Aten Prima - 1995; 421-5.

11. Saturno PJ. Los métodos de participación del usuario en la evaluación y mejora de la calidad de los servicios sanitarios. Rev Esp Salud Pública. 1995; 69:163-75.

12. Crow R, Gage H, Hampson $S$ et al. The measurement of satisfaction with healthcare: implications for practise from a systematic review of the literature. Health Technol Assess. 2002; 6:1-245.

13. Martín Arribas MC. Diseño y validación de cuestionarios. Matronas Profesión, 2004, vol 5, num 17. 2004; 5.

14. Salomón L, Gasquet I, Mesbah M, Ravaud P. Construction of a scale measuring impatients' opinion on quality of care. Int J Qual Health Care; 11: 507-516. 1999.

15. Labarere J, Francois P, Auquier P, Robert C, Fourny M. Development of a French impatient satisfaction questionnaire. Int J Qual Health Care. 2001; 13:99-108.

16. Hardy GE, West MA, Hill F. Validation of a questionnaire measuring patient satisfaction with general practitioner services -- Grogan et al. 9 (4): 210 -- BMJ Quality and Safety. 2000. Disponible en: http://qualitysafety. bmj.com/content/9/4/210.short.
17. Cronin JJ, Jr., Taylor SA. Measuring Service Quality: A Reexamination and Extension. Journal of Marketing. 1992; 56(3):55-68.

18. Cronin Jr JJ, Taylor SA. SERVPERF versus SERVQUAL: Reconciling performance-based and perceptions-minus-expectations measurement of service quality. The Journal of Marketing. 1994; 125-131.

19. Díaz de Rada Igurquiza V. Análisis Factorial. Técnicas de análisis multivariante para investigación social y comercial. Madrid: RA-MA; 2002. p. 91-156

20. Cronbach LJ. Coefficient alpha and the internal structure of test. Psychometrika. 1951; 16: 297334.

21. Gené-Badia J, Ascaso C, Escaramis-Babiano G, Sampietro-Co- Iom L, Catalán-Ramos $A$, Sans-Corrales $M$, et al. Personalised care, access, quality and team coordination are the main dimensions of family medicine output. Fam Pract. Oxford Journals Medicine \& Health. 2007; vol 24:41-7.

22. Pasarín MI, Berra S, Rajmil L, Solans M, Borrell C, Starfield B. Un instrumento para la evaluación de la atención primaria de salud desde la perspectiva de la población. Aten Primaria. agosto de 2007; 39:395-401.

23. Landeta, Jon. El método Delphi: una técnica de previsión para la incertidumbre. Barcelona: Ariel, 1999.

24. Batista-Foguet JM, Coenders G, Alonso J. Análisis factorial confirmatorio. Su utilidad en la validación de cuestionarios relacionados con la salud. Med Clin (Barc) 2004; 122: 21-27. 2004.

25. Batista-Foguet JM, Coenders G. Introducción a los modelos estructurales. Utilización del análisis factorial confirmatorio para la depuración de un cuestionario. En: Renom J, editor. Tratamiento informatizado de datos. Barcelona: Masson, 1998; p. 22.

26. Oviedo Sarmiento E. Nivel de satisfacción de las pacientes con y sin preparación en psicoprofilaxis obstétrica al recibir la atención del primer parto eutócico en el Hospital I Jorge Voto Bernales Corpancho: EsSalud. Lima, Perú. [Tesis Maestría]. [Lima - Perú]: Universidad Nacional Mayor de San Marcos; 2010.

27. Lora Loza M.G. Calidad de atención materna en los servicios de Obstetricia percibido por las usuarias: estudio comparativo en dos Hospitales Regionales del Norte del Perú. [Lima - Perú]: Universidad Nacional Mayor de San Marcos; 2006.

28. Wajajay Nuñez W. Percepción de la calidad de atención del parto en usuarias del servicio de centro obstétrico del Hospital Nacional Daniel Alcides Carrión. Enero - Febrero 2013. [Lima - Perú]: Universidad Nacional Mayor de San Marcos.

29. Espinoza Rojas J, Percepción de la calidad de atención del parto en usuarias del Centro Obstétrico del Hospital San Juan de Lurigancho enero 2014. [Lima - Perú]: Universidad Nacional Mayor de San Marcos; 2014

30. Miranda Benavente y Col. Calidad de atención del parto vaginal en gestantes del distrito Gregorio Albarracin atendidas en los servicios públicos de salud, Tacna - 2006 